\title{
Feasibility of Laparoscopic Proximal Gastrectomy With Piggyback Jejunal Interposition Double-Tract Reconstruction for Proximal Gastric Cancer. A Propensity Score Matching Analysis
}

\author{
ZhiGuo Li \\ Shanxi Cancer Hospital \\ JianHong Dong ( $\nabla$ asdjh666@163.com ) \\ Shanxi Cancer Hospital \\ QingXing Huang \\ Shanxi Cancer Hospital \\ Wei Guo \\ Heji Hospital, Affiliated to Changzhi Medical College \\ YanYang Song \\ Yuncheng Central Hospital \\ WanHong Zhang \\ Shanxi Cancer Hospital \\ Jun Ma \\ Shanxi Cancer Hospital \\ Yi Wang \\ Shanxi Cancer Hospital \\ GuoLong Ma \\ Shanxi Cancer Hospital \\ ZhenHua Wang \\ Shanxi Cancer Hospital \\ YiPeng Ren \\ Shanxi Cancer Hospital \\ Fei Gao \\ Shanxi Cancer Hospital \\ LinJie Li \\ Shanxi Cancer Hospital
}

Research Article 
Keywords: Proximal gastrectomy, Total gastrectomy, Laparoscopic, Double-tract, Proximal gastric cancer, Reconstruction, Piggyback jejunal interposition

Posted Date: September 22nd, 2021

DOI: https://doi.org/10.21203/rs.3.rs-882462/v1

License: (1) This work is licensed under a Creative Commons Attribution 4.0 International License. Read Full License 


\section{Abstract \\ Background}

The clinical effect of laparoscopic proximal gastrectomy (LPG) with piggyback jejunal interposition double-tract reconstruction (PJIDTR) is not clear yet. The purpose of this study was to compare the efficacy of LPG with PJIDTR versus laparoscopic total gastrectomy (LTG) with Roux-en-Y reconstruction (overlap method) for proximal EGC using propensity score matching.

\section{Methods}

We retrospectively collected data from the patients with proximal EGC who were treated in Shanxi Cancer Hospital between January 2012 and December 2015. Propensity score was used to match LTG patients with LPG patients. The patient characteristics, clinical outcomes, nutritional indicators, reflux esophagitis incidence and overall survival were compared between the two groups.

\section{Results}

Of the 424 patients, 200 were excluded and 50 of the remaining patients received LPG with PJIDTR. Fifty matched LTG patients were screened. Comparison of intraoperative indicators, operative time, blood loss and number of retrieved lymphnodes between the two groups showed no statistical significance $(P>$ $0.05)$. There was no statistically significant differences in first anal exhaust time, postoperative hospital stay, and early complications between the two groups $(P>0.05)$. The nutrition indexes at one year after surgery in the LPG group were significantly better than LTG group $(P<0.05)$. At one year, there were no differences in reflux symptoms (Visick score) or endoscopic esophagitis (Los Angeles Classification) between the two groups. No tumor recurrence was observed in either group. The 5-year overall survival rates of the two groups were $98 \%$ and $90 \%$, respectively $(p=0.08)$.

\section{Conclusion}

The postoperative nutritional outcomes of LPG with PJIDTR were better than those of LTG. The incidence of reflux esophagitis, complications, and 5-year overall survival were similar between the two groups. LPG with PJIDTR may be suitable for proximal EGC.

\section{Introduction}

In recent years, the incidence of proximal gastric cancer is increasing. Globocan data show that in 2012, there were 952,000 new cases of gastric cancer in the world, among which 260,000 cases of cardiac cancer, $52 \%$ (135,000 cases) were in China [1]. Among 7887 patients with gastric cancer admitted to 
Shanxi Cancer Hospital from 2011 to 2016, proximal gastric cancer accounted for 40.4\% [2]. In Korea, the incidence of proximal gastric cancer increased from $11.2 \%$ in 1995 to $16.0 \%$ in 2014 [3].

Proximal gastrectomy (PG) for proximal early gastric cancer (EGC) has been widely accepted in Japan and South Korea, as it provides the same overall survival as total gastrectomy (TG)[4], But it gives the patient a better ability to eat and a better quality of life [5-7]. In contrast, TG is routinely performed for proximal EGC and advanced esophagogastric junction carcinoma in western countries [4]. PG is recommended when more than half of the stomach is preserved, as many studies have shown better nutritional status after PG than after TG [8-11]. Because there was no difference in survival between PG and TG, patients with extensive lymphadenectomy did not benefit [12]. A 2019 retrospective analysis of 202 upper 1/3 gastric cancer patients with pT2/T3 staging in Japan showed, the rate of lymph node metastasis was very low in the No.4d and No.12a. However, the rate of lymph node metastasis was zero in No.5 and No.6. These results suggest that PG is a safe option for upper $1 / 3$ gastric cancer with T2/T3 stage.

The development of laparoscopic technology makes laparoscopic gastrectomy gradually applied in clinic [13-16]. The wide application of LPG in clinic has not been accepted [17-18]. According to a nationwide survey conducted by the Japan Endoscopic Surgery Society (JSES), about 2,800 gastric cancer patients underwent LPG surgery from 1994 to 2013. The proportion of LPG surgery increased gradually. In 2013, the number of LPG was not only higher than Open PG, but also accounted for $4.6 \%$ of the total laparoscopic gastric cancer surgeries in Japan [19]. Although there is not enough evidence-based medical evidence to prove that laparoscopic surgery is superior to open surgery in the treatment of proximal EGC, Japan has made some progress in LPG.

The purpose of this retrospective cohort study was to compare LPG with piggyback jejunal interposition double-tract reconstruction (PJIDTR) [20] versus LTG with Roux-en-Y reconstruction (overlap method) in terms of oncological safety, functional benefit, complications and reflux esophagitis in patients with proximal EGC.

\section{Patients And Methods}

\section{Ethics Statement}

This study was a retrospective cohort study. This study was approved by the Ethics Committee of Shanxi Cancer Hospital (approval no.202028). All participants signed a written informed consent and all methods were performed in accordance with the Declaration of Helsinki.

\section{Patients}

The clinical data of patients who underwent laparoscopic upper gastric tumor resection in Shanxi cancer hospital from January 2017 to December 2015 were collected retrospectively. In this study, upper gastric cancer refers to the tumor located in the upper $1 / 3$ of the stomach after the tripartite division of greater 
curvature and lesser curvature. The inclusion criteria included: 1) Preoperative endoscopic and pathological diagnosis was confirmed as gastric cancer, and all were early cancers; 2) Age $\geq 18$ years; 3 ) All operations were performed under microscope, and the postoperative pathological diagnosis was R0 resection; 3 ) tumor size < $4 \mathrm{~cm}$; 4) No history of abdominal surgery; The exclusion criteria included: 1 ) Lack of clinical data; 2) Emergency surgery or switch to open surgery; 3 ) Clinical advanced stage >cT2;4) Age $₫ 18$ years; 5) Esophageal invasion.

Patients were divided into two groups, LPG group and LTG group. Patients with R1 resection were excluded from this study due to statistically meaningless. Propensity score matching analysis was performed according to age, sex, body mass index (BMI), American Society of Anesthesiologists (ASA) score, degree of differentiation and pathological stage (Table 1). According to 1:1 propensity matching, 100 patients were finally included (Fig. 1).

\section{Surgical Procedure}

All surgeries were performed by the same team of surgeons with extensive laparoscopic experience.

\section{Laparoscopic proximal gastrectomy}

We adopted the surgical procedures of Li ZG et al. [20]. The LPG procedures were performed by the 5-port technique under general anesthesia. The patient was supine with legs divided, the cephalic side slightly higher, about 15 degrees. After thoroughly examining the internal organs, dissection of D1 + lymph nodes for proximal gastric cancer was performed, and the range of No.1, 2, 3a, 4sa, 4sb, 7, 8a, 9 and 11p lymphnodes were included in the proximal stomach (about 2/3). The digestive tract reconstruction process is as follows. The gastric wall was cut off in the vascularless region of the greater curvature of the stomach with a laparoscopic linear cutting and closure device (ETHICON, EC60A, ECR60B), and more than half of the residual stomach was preserved. Ligature band was used $1 \mathrm{~cm}$ above the cardia and led caudally. An incision of $1 \mathrm{~cm}$ was made in the left anterior wall of the esophagus and the jejunum wall $20 \mathrm{~cm}$ from Treitz. The laparoscopic linear cutting and closing device (ETHICON, EC60A, ECR60B) was extended into the esophageal cavity and the jejunum cavity respectively, and the esophagojejunal anastomosis was performed side-to-side. The laparoscopic linear cutting and closing device (ETHICON, EC60A, ECR60B) closes the common opening while the PG specimen is resected. A $1 \mathrm{~cm}$ incision was made in the jejunum wall and the anterior wall of the greater curvature of the remnant stomach at a distance of $12-15 \mathrm{~cm}$ from the esophagojejunostomy, respectively. A laparoscopic linear cutting closure device (ETHICON, EC60A, ECR60B) was inserted into the two incisions to perform the side-to-side gastrojejunostomy, and then the common opening was closed. An incision of $1 \mathrm{~cm}$ was made in the near broken end of jejunum and the jejunum wall $30 \mathrm{~cm}$ away from the distal end of the gastrojejunum anastomosis, and a laparoscopic linear cutting closure device (ETHICON, EC60A, ECR60B) was inserted to perform jejunum-jejunum side-to-side anastomosis. Check that the anastomosis is satisfactory, and then close the common opening. The specimen was subsequently removed through the umbilical port site by extending the incision.

\section{Laparoscopic total gastrectomy}


The patient's anesthesia, body position and working ports position should be the same as LPG. After thoroughly examining the internal organs, dissection of D1 + lymph nodes for total gastric cancer was performed, and the range of No.1, 2, 3, 4sa, 4sb, 4d, 5, 6, 7, 8a, 9 and 11p lymph nodes were included in the total stomach. Roux-en-Y esophagojejunostomy (overlap method) was performed after the gastrectomy. After the abdominal esophagus was fully nude and dissociated, it was rotated $90^{\circ}$ clockwise. The esophagus was separated through the main operating hole with a laparoscopic linear cutting and closing device (ETHICON, EC60A, ECR60B), and the cut line of the esophagus was sagittal. Jejunum was separated $20 \mathrm{~cm}$ from Treitz with a linear cutting and closing device (ETHICON, EC60A, ECR60B), and jejunum was anastomosed side-to-side between the proximal jejunum and the distal jejunum $35 \mathrm{~cm}$ from the distal jejunum. Suture was pulled at the $5 \mathrm{~cm}$ mesangium of the distal jejunum, and a hole was opened at the distal end to make a loop for anastomosis. A hole was made on the dorsal side of the broken end of the esophagus and traction was performed. The nail and non-nail arms of the linear cutting and closing device (ETHICON, EC60A, ECR60B) were placed into the jejunum and esophagus respectively, and side to side anastomosis was performed. Check that the anastomosis is satisfactory, and then close the common opening. The specimen was subsequently removed through the umbilical port site by extending the incision.

\section{Clinical Analysis and Surgical Outcomes}

The patients were followed up five years after surgery. The patients were followed up by telephone and returned to the hospital regularly for review. The following data were recorded and analyzed: age, sex, BMI, American Society of anesthesiologists (ASA) score, tumor differentiation, pathological stage based on AJCC / UICC 8th Edition [21], operation time, blood loss and number of retrieved LNs. The postoperative evaluation indexes were the first anal exhaust time, postoperative hospital stay and early complications. Follow-up outcomes were nutrition indexes include BMI, hemoglobin, total serum protein and serum albumin. Visick score [22] was used to evaluate gastrophageal reflux symptoms one year after surgery: Visick grade I, asymptomatic; Visick grade II, occasional symptoms; Visick grade III, apparent but tolerable symptoms; and Visick grade IV, apparent and intolerable symptoms. All patients underwent postoperative endoscopy to evaluate gastroesophageal reflux according to the Los Angeles Classification System [23]. Death and cause of death were determined through follow-up and cause of disease.

\section{Statistical Analysis}

All statistical analysis were performed with SPSS software version 26.0 for Windows (SPSS, Chicago, IL,USA). The LPG and LTG groups were compared in terms of continuous variables using Student's t test and in terms of categorical variables using the chi-squared test or Fisher's exact test. The Kaplan-Meier method was used for calculating survival rate. The difference between the curves was assessed using the log-rank test. $P \otimes 0.05$ was considered statistically significant.

\section{Results}


424 patients with proximal gastric cancer who received surgical treatment were included in the study, and 200 of them were excluded. Exclusions are as follows: 80 cases of clinical advanced stage >cT2, 90 cases lacked clinical data, 20 patients had esophageal invasion, 7 cases were switched to open surgery or emergency surgery, 3 cases of age $<18$ years. The remaining 224 patients with proximal EGC were screened by admittance criteria, 163 patients underwent LTG with Roux-en-Y reconstruction (overlap method) and 50 patients underwent LPG with PJIDTR (Fig. 1).

Baseline characteristics of two groups were well balanced $(P>0.05)$. Comparison of intraoperative indicators, operative time, blood loss and number of retrieved lymphnodes between the two groups showed no statistical significance $(P>0.05)$ (Table 1$)$. 
Table 1

Patient characteristics

\section{LPG with PJIDTR( $n=50) \quad$ LTG with RY(Over lap) $(n=50) \quad P$ value}

$\operatorname{Sex}, \mathrm{n}(\%)$

Male

Female

Age, years

$\mathrm{BMI}, \mathrm{kg} / \mathrm{m}^{2}$

ASA score

।

II

III

Differentiation,n (\%)

High-middle

Low

pStage,n (\%)

IA

IB

Operative time,min

Blood loss, $\mathrm{mL}$

Number of retrieved LNs

$25.7 \pm 4.6$

$24.1 \pm 1.8$
$40(80 \%)$

$10(20 \%)$

$66.9 \pm 3.6$

0.13

$23.8 \pm 2.0$

0.26

$15(30 \%)$

0.83

$34(68 \%)$

$1(2 \%)$

0.62

$39(78 \%)$

$11(22 \%)$

0.56

49(98\%)

$1(2 \%)$

$187.4 \pm 6.7$

0.38

$117.0 \pm 51.3$

0.37

$34.4 \pm 5.2$

0.51

LPG, Laparoscopic proximal gastrectomy; LTG, Laparoscopic total gastrectomy; PJIDTR, piggyback jejunal interposition double-tract reconstruction; BMI, Body mass index; ASA, American Society of Anesthesiologists; pStage, pathological stage; LN, Lymphnode; RY, Roux-en-Y

Postoperative recovery indexes: the first anal exhaust time, postoperative hospital stay and early complications (within 1 month after operation) had no significant difference $(P>0.05)$ (Table 2$)$. 
Table 2

Postoperative outcomes

\begin{tabular}{|llll|}
\hline & LPG with PJIDTR(n= & LTG with RY(Over lap)(n= 50) & Pvalue \\
\hline First anal exhaust time(h) & $4.2 \pm 0.4$ & $4.1 \pm 0.3$ & 0.06 \\
\hline $\begin{array}{l}\text { Postoperative hospital } \\
\text { stay(d) }\end{array}$ & $14.1 \pm 4.4$ & $14.6 \pm 6.0$ & 0.10 \\
\hline Early complications & $7(14 \%)$ & $8(16 \%)$ & 0.56 \\
\hline Anastomotic leakage & 1 & 1 & \\
\hline Anastomotic bleeding & 2 & 1 & \\
\hline Anastomotic stricture & 0 & 0 & \\
\hline Abdominal infection & 1 & 1 & \\
\hline Incision infection & 1 & 1 & \\
\hline Ileus & 2 & 1 & \\
\hline Pancreatic fistula & 0 & 1 & \\
\hline Dumping syndrome & 0 & 0 \\
\hline Respiratory & 0 & 2 \\
\hline LPG, Laparoscopic proximal gastrectomy; LTG, Laparoscopic total gastrectomy; PJIDTR, piggyback \\
jejunal interposition double-tract reconstruction; RY, Roux-en-Y
\end{tabular}

As shown in Table 3, there were significant differences in BMI, hemoglobin, total serum protein and serum albumin between the two groups one year after operation $(P<0.05)$. Visick scoring of the reflux symptoms one year after surgery showed that one patient in the LPG group and two patients in the LTG group had grade II reflux. These patients did not appeal any further reflux symptoms at the time of the next follow-up (Table 4). 
Table 3

Nutrition indexes

\section{LPG with PJIDTR( $=50) \quad$ LTG with RY(Over lap) $(n=50) \quad P$ value}

$\mathrm{BMI}$

$\begin{array}{llll}\text { Preoperative } & 24.1 \pm 1.8 & 23.8 \pm 2.0 & 0.26 \\ \text { Postoperative one year } & 22.9 \pm 2.0 & 20.6 \pm 1.3 & 0.01\end{array}$

Hemoglobin

$\begin{array}{llll}\text { Preoperative } & 116.9 \pm 13.4 & 114.5 \pm 12.4 & 0.36 \\ \text { Postoperative one year } & 122.4 \pm 6.0 & 112.7 \pm 7.3 & 0.00\end{array}$

Total serum protein

$\begin{array}{llll}\text { Preoperative } & 68.4 \pm 5.4 & 67.1 \pm 5.6 & 0.26 \\ \text { Postoperative one year } & 69.8 \pm 4.8 & 67.0 \pm 5.2 & 0.01\end{array}$

Serum albumin

$\begin{array}{llll}\text { Preoperative } & 43.4 \pm 3.1 & 43.0 \pm 3.6 & 0.55 \\ \text { Postoperative one year } & 43.0 \pm 3.7 & 41.4 \pm 2.7 & 0.02\end{array}$

LPG, Laparoscopic proximal gastrectomy; LTG, Laparoscopic total gastrectomy; PJIDTR, piggyback jejunal interposition double-tract reconstruction; RY, Roux-en-Y; BMI, Body mass index

Table 4

Reflux symptom scores one year after surgery

\begin{tabular}{|lll|}
\hline Visick score & LPG with PJIDTR $(\mathbf{n}=\mathbf{5 0})$ & LTG with RY(Over lap)(n= 50) \\
\hline I & 49 & 48 \\
\hline II & 1 & 2 \\
\hline III & 0 & 0 \\
\hline IV & 0 & 0 \\
\hline LPG, Laparoscopic proximal gastrectomy; LTG, Laparoscopic total gastrectomy; PJIDTR, piggyback \\
jejunal interposition double-tract reconstruction; RY, Roux-en-Y
\end{tabular}

As shown in Table 5, endoscopy was performed on all patients one year after surgery. Preoperative and postoperative endoscopic scores of all patients were determined according to Los Angeles Classification of reflux esophagitis. In the LPG group, there were five grade A and one grade B reflux esophagitis preoperatively. There were two grade A reflux esophagitis postoperatively in the LPG group. In the LTG 
group, there were four grade $A$ and one grade $B$ reflux esophagitis preoperatively. There were three grade $A$ and one grade $B$ reflux esophagitis postoperatively in the LTG group.

Table 5

Pre- and postoperative reflux esophagitis scores

\begin{tabular}{|lllll|}
\hline LA grade & LPG with PJIDTR(n= 50) & \multicolumn{2}{l|}{ LTG with RY(Over lap)( $\mathbf{n = 5 0 )}$} \\
\hline Grade & Preoperative & Postoperative & Preoperative & Postoperative \\
\hline A & 5 & 2 & 4 & 3 \\
\hline B & 1 & 0 & 1 & 1 \\
C & 0 & 0 & 0 & 0 \\
\hline D & 0 & 0 & 0 & 0 \\
\hline $\begin{array}{l}\text { LPG, Laparoscopic proximal gastrectomy; LTG, Laparoscopic total gastrectomy; PJIDTR, piggyback } \\
\text { jejunal interposition double-tract reconstruction; RY, Roux-en-Y; LA: Los Angeles }\end{array}$ \\
\hline
\end{tabular}

One patient in the LPG group died from cerebral infarction. In the LTG group, two died of respiratory failure and three died of heart disease. None of the above deaths was directly related to gastric cancer. During follow-up, no tumor recurrence was found in both groups. As shown in Fig. 2, the Log Rank test result of the overall comparison of the survival curves of the two groups was $P=0.08$. No significant difference in survival between the two groups.

\section{Discussion}

The incidence rate of proximal gastric cancer has increased significantly in the world in the past 20 years. $[24,25]$. The choice and safety of excision methods for upper gastric carcinoma have become one of the hot topics in research. TG with D2 lymphadenectomy has become the standard surgical procedure for patients with advanced proximal gastric cancer. Patients with EGC usually have long postoperative survival and low recurrence rate [26]. Therefore, it is very important for EGC patients to ensure their postoperative quality of life [27]. Multiple studies have shown that patients who undergo radical PG have a better quality of life after surgery than those who undergo TG [28-30]. The Japanese Gastric Cancer Treatment Guidelines suggest that PG should be used in patients with proximal EGC, and the residual stomach should be retained at least $50 \%$ [8]. Usually, the residual stomach of $12 \mathrm{~cm}$ or more along the lesser curvature and $25 \mathrm{~cm}$ or more along the greater curvature from the pylorus ring is preserved [2].

With the development of laparoscopic instruments and the progress of technology, the application of laparoscopy in proximal gastric cancer is bound to be one of the key directions in the future. Our previous study reported the short-term efficacy of open PG with PJIDTR [20]. In particular, the efficacy of LPG with PJIDTR remains unclear due to the lack of short and long-term studies. 
The application of PG to proximal EGC has been limited due to the following four main concerns: oncologic safety, nutrition benefits, gastroesophageal reflux and anastomotic stenosis. The results showed that there was no significant difference between the two groups in operation time, bleeding volume, first anal exhaust time and postoperative hospital stay. Although LPG with PJIDTR requires three anastomoses and the procedures seem to be more complicated, there was no significant difference in anastomotic complications between the two surgical methods (6\% vs. $4 \%$ ) (Table 2). Stricture of the residual gastrojejunal anastomosis after LPG with DTR will lead to food flow without passing through the residual stomach. Ahn SH et al. [31] performed a routine gastric emptying scan at three months after the operation. The relative ratio of food flow from the stomach to the small intestine after DTR was 3:2. Yamashita $\mathrm{K}$ et al. [32] evaluated the actual food flow one year after DTR by digestive tract radiography for the first time, and $17.5 \%$ of the patients had food directly entering the small intestine without passing through the residual stomach. Presumably, there are several reasons why food doesn't pass through the residual stomach. First, the retained residual stomach volume is too small, resulting in the residual stomach pressure is greater than the jejunal route; Second, the direction and size of the residual gastrojejunal anastomosis lead to easier food flow into the jejunum; Third, gastrojejunal anastomotic stenosis, causing food to be unable to enter the residual stomach. No relevant anastomotic stenosis was found in our study because of the large side-to-side anastomosis and and more than half of the gastric remnant.

When more than half stomach can be preserved, the PG is chosen, because the nutritional status after the PG were shown to be better than after the TG by many studies [9-11]. According to PGSAS, PG was superior to TG in weight loss, dietary supplementation, diarrhea and dumping syndrome [33]. The reason is that the presence of gastric remnant reduces the incidence of angastric anemia due to vitamin B12 and iron absorption disorders. The presence of pylorus reduces the incidence of bile reflux and dumping syndrome. As this study shows (Table 3), the nutrition indexes at one year after surgery in the LPG group were significantly better than LTG group $(P<0.05)$. LPG was superior to LTG in controlling skeletal muscle loss, maintaining stable body weight, reducing anemia, and improving quality of life.

In this study, we analyzed the surgical outcomes of LPG with PJIDTR in 50 patients with proximal EGC. To our knowledge, this is first report to describe the application of LPG with PJIDTR for proximal EGC, which shows good postoperative effects, especially the reduction of reflux symptoms. Only one patient had grade II reflux symptoms in LPG group. This good result reflects the fact that PJIDTR in LPG associates with fewer reflux symptoms than LPG with other reconstruction methods. Several previous studies have applied direct esophagogastric anastomosis as the reconstruction method, probably because it is simple and needs only one anastomosis. Many anti-reflux procedures are used to prevent reflux esophagitis, such as gastric tube formation, fundoplication, esophagopexy with crural repair and pyloroplasty. However, all these methods involved esophagogastrostomy, and the results were disappointing since the rate of reflux esophagitis were still high [34-36]. A good alternative to esophagogastrostomy reconstruction after PG is the PJIDTR, which is very effective anti-reflux reconstruction. At our institution, LPG with PJIDTR was also performed since February 2010 and showed a low rate of early postoperative complications, especially anti-reflux effect and nutritional recovery [20]. 
We found that the oncological safety of LPG with PJIDTR was satisfactory: no tumor recurrence was observed. It should be noted that most of the five LTG patients who died were elderly with underlying cardiopulmonary disease. There was no significant difference in survival rate between the two groups. The results are consistent with relevant studies $[4,12]$. Systematic meta-analysis compared TG and PG, it was concluded that TG and PG had similar overall survival outcomes for proximal gastric cancer [4].

This retrospective cohort study had the following three limitations. First, retrospective studies have a lower level of evidence than prospective studies. Second, we didn't assess the quality of life of the patients because it was not fully followed up by using a validated questionnaire, Third, the sample size of this study can be further expanded. However, to our knowledge, this is the first retrospective study of LPG with PJIDTR. These encouraging data lead us to plan phase III multicenter prospective randomized clinical trial about LPG with PJIDTR.

\section{Conclusions}

This study showed that, in proximal EGC, LPG with PJIDTR was better than LTG with Roux-en-Y reconstruction in terms of nutrition outcomes. This suggests that LPG with PJIDTR may be suitable surgical option for proximal EGC.

\section{Abbreviations}

EGC: Early gastric cancer; PG: Proximal gastrectomy; TG: Total gastrectomy; LPG: Laparoscopic proximal gastrectomy; LTG: Laparoscopic total gastrectomy; JSES: Japan Society for Endoscopic Surgery; DTR: Double-tract reconstruction; PJIDTR: piggyback jejunal interposition double-tract reconstruction; BMl: Body mass index; ASA: American Society of Anesthesiologists; pStage: pathological stage; LN: Lymphnode; EJ: Esophagojejunostomy; RY: Roux-en-Y; LA: Los Angeles.

\section{Declarations}

\section{Ethics approval and consent to participate}

This study was approved by the ethics committee of Shanxi Cancer Hospital (approval no.202028). All patients signed informed consent and all methods were performed in accordance with the Declaration of Helsinki.

\section{Consent for publication}

Not applicable.

\section{Availability of data and materials}

The data used to support the findings of this study are available from the corresponding author upon request. 


\section{Competing interests}

The authors declare that they have no conflict of interest.

\section{Funding}

This study was supported by "Four batches" science and technology to revitalize the medical innovation project (no.2020XM48) and Key Research and Development (R\&D) Projects of Shanxi Province (no.201803D31167).

\section{Authors' contributions}

Study conception and design: ZL, JD and YS. Acquisition, analysis, and interpretation of data: ZL, QH, WG, WZ, YW, GM, ZW, YR, FG, and LL. Drafting of manuscript or critical revision: ZL, JD and JM. All authors read and approved the final version of the manuscript.

\section{Acknowledgements}

Not applicable.

\section{References}

1. A Colquhoun, M Arnold, J Ferlay, K J Goodman, D Forman, I Soerjomataram. Global patterns of cardia and non-cardia gastric cancer incidence in 2012. Gut. 2015;64(12):1881-8.

2. Li ZG, Dong JH, Huang QX, Tao K, Ma J, Zhang WH. Proximal gastrectomy and digestive tract reconstruction: status survey of perceptions and treatment selection in Chinese surgeons. Chinese journal of gastrointestinal surgery. 2020;23(8):757 - 65(in Chinese).

3. Information Committee of Korean Gastric Cancer Association. Korean Gastric Cancer Association Nationwide Survey on Gastric Cancer in 2014. J Gastric Cancer. 2016;16(3):131 - 40.

4. Wen L, Chen XZ, Wu B, Chen XL, Wang L, Yang K, Zhang B, Chen ZX, Chen JP, Zhou ZG, Li CM, Hu JK. Total vs. proximal gastrectomy for proximal gastric cancer: a systematic review and meta-analysis. Hepatogastroenterology. 2012;59(114):633 - 40.

5. Adachi Y, Inoue T, Hagino Y, Shiraishi N, Shimoda K, Kitano S. Surgical results of proximal gastrectomy for early-stage gastric cancer: jejunal interposition and gastric tube reconstruction.Gastric Cancer. 1999;2(1):40 - 5.

6. Takeshita K, Saito N, Saeki I, Honda T, Tani M, Kando F, Endo M. Proximal gastrectomy and jejunal pouch interposition for the treatment of early cancer in the upper third of the stomach: surgical techniques and evaluation of postoperative function. Surgery.1997;121(3):278 - 86.

7. Kameyama J, Ishida H, Yasaku Y, Suzuki A, Kuzu H, Tsukamoto M. Proximal gastrectomy reconstructed by interposition of a jejunal pouch. Surgical technique. The European journal of surgery. 1993;159(9):491-3. 
8. Japanese Gastric Cancer Association; Japanese gastric cancer treatment guidelines 2018 (5th edition).Gastric cancer: official journal of the International Gastric Cancer Association and the Japanese Gastric Cancer Association. 2021;24(1):1-21.

9. Huh YJ, Lee HJ, Oh SY, Lee KG, Yang JY, Ahn HS, Suh YS, Kong SH, Lee KU, Yang HK.Clinical outcome of modified laparoscopy-assisted proximal gastrectomy compared to conventional proximal gastrectomy or total gastrectomy for upper-third early gastric cancer with special references to postoperative reflux esophagitis. J Gastric Cancer. 2015;15(3):191-200.

10. Nishigori T, Okabe H, Tsunoda S, Shinohara H, Obama K, Hosogi H, Hisamori S, Miyazaki K, Nakayama T, Sakai Y. Superiority of laparoscopic proximal gastrectomy with hand-sewn esophagogastrostomy over total gastrectomy in improving postoperative body weight loss and quality of life. Surg Endosc. 2017;31(9):3664-72.

11. Jung DH, Lee Y, Kim DW, Park YS, Ahn SH, Park DJ, Kim HH. Laparoscopic proximal gastrectomy with double tract reconstruction is superior to laparoscopic total gastrectomy for proximal early gastric cancer. Surg Endosc. 2017;31(10):3961-9.

12. Sugoor $P$, Shah S, Dusane R, Desouza A, Goel M, Shrikhande SV. Proximal gastrectomy versus total gastrectomy for proximal third gastric cancer: total gastrectomy is not always necessary. Langenbeck's Arch Surg. 2016;401(5):687 - 97.

13. Shiraishi N, Yasuda K, Kitano S. Laparoscopic gastrectomy with lymph node dissection for gastric cancer. Gastric Cancer. 2006;9(10):167 - 76.

14. Cai J, Wei D, Gao CF, Zhang CS, Zhang $\mathrm{H}$, Zhao T. A prospective randomized study comparing open versus laparoscopy-assisted D2 radical gastrectomy in advanced gastric cancer. Dig Surg. 2011;28(5-6):331-7.

15. Hu Y, Ying M, Huang C, Wei H, Jiang Z, Peng X, Hu JK, Du XH, Wang BL, Lin F, Xu J, Dong GL, Mou TY, Li GX, Chinese Laparoscopic Gastrointestinal Surgery Study (CLASS) Group. Oncologic outcomes of laparoscopy-assisted gastrectomy for advanced gastric cancer: a large-scale multicenter retrospective cohort study from China. Surg Endosc. 2014;28(7):2048-56.

16. Fang C, Hua J, Li J, Zhen J, Wang F, Zhao Q, Shuang JB, Du JJ. Comparison of long-term results between laparoscopy-assisted gastrectomy and open gastrectomy with D2 lymphadenectomy for advanced gastric cancer. Am J Surg. 2014;208(3):391-6.

17. Tanimura S, Higashino M, Fukunaga Y, Takemura M, Tanaka Y, Fujiwara Y, Osugi H. Laparoscopic gastrectomy for gastric cancer: experience with more than 600 cases. Surg Endosc. 2008;22(5):11614.

18. Kitano S, Shiraishi N, Uyama I, Sugihara K, Tanigawa N, Japanese Laparoscopic Surgery Study Group. A multi-center study on oncologic outcome of laparoscopic gastrectomy for early cancer in Japan. Ann Surg. 2007;245(1):68-72.

19. Japan Society for Endoscopic Surgery. Nationwide survey on endoscopic surgery in Japan. J Jpn Soc Endosc Surg. 2014;5:535 - 40 (in Japanese). 
20. Li ZG, Dong JH, Huang QX, Zhang WH, Tao K. Comparison of three digestive tract reconstruction methods for the treatment of Siewert II and III adenocarcinoma of esophagogastric junction: a prospective, randomized controlled study. World journal of surgical oncology. 2019;17(1):209.

21. Brierley JD, Gospodarowicz MK, Wittekind C. TNM Classifcation of Malignant Tumours (8th ed.). Wiley Blackwell. 2016. https://www.wiley.com/en$\mathrm{gb} / \mathrm{TNM}+$ Classification+of+Malignant+Tumours\%2C+8th+Edition-p-9781119263579.

22. Visick AH. A study of the failures after gastrectomy. Annals of the Royal College of Surgeons of England. 1948;3(5):266 - 84.

23. Kubo M, Sasako M, Gotoda T, Ono H, Fujishiro M, Saito D, Sano T, Katai H. Endoscopic evaluation of the remnant stomach after gastrectomy: proposal for a new classification. Gastric cancer:official journal of the International Gastric Cancer Association and the Japanese Gastric Cancer Association. 2002;5(2):83 - 9 .

24. Ahn HS, Lee HJ, Yoo MW, Jeong SH, Park DJ, Kim HH, Kim WH, Lee KU, Yang HK. Changes in clinicopathological features and survival after gastrectomy for gastric cancer over a 20-year period. Br J Surg. 2011;98(2):255 - 60.

25. Sehdev A, Catenacci DV. Gastroesophageal cancer: focus on epidemiology, classification, and staging. Discov Med. 2013;16(87):103 - 11.

26. Ikeguchi M, Kader A, Takaya S, Fukumoto Y, Osaki T, Saito H, Tatebe S, Wakatsuki T. Prognosis of patients with gastric cancer who underwent proximal gastrectomy. International surgery. 2012;97(3):275-9.

27. Hiki N, Nunobe S, Kubota T, Jiang XH. Function-preserving gastrectomy for early gastric cancer. Ann Surg Oncol. 2013;20(8):2683-92.

28. Hosoda K, Yamashita K, Katada N, Moriya H, Mieno H, Shibata T, Sakuramoto S, Kikuchi S, Watanabe M. Potential benefits of laparoscopy-assisted proximal gastrectomy with esophagogastrostomy for cT1 upper-third gastric cancer. Surg Endosc. 2016;30(8):3426-36.

29. Masuzawa T, Takiguchi S, Hirao M, Imamura H, Kimura Y, Fujita J, Miyashiro I, Tamura S, Hiratsuka M, Kobayashi K, Fujiwara Y, Mori M, Doki Y. Comparison of perioperative and long-term outcomes of total and proximal gastrectomy for early gastric cancer: a multi-institutional retrospective study. World J Surg. 2014;38(5):1100-06.

30. Nomura E, Kayano H, Lee SW, Kawai M, Machida T, Yamamoto S, Nabeshima K, Nakamura K, Mukai $\mathrm{M}$, Uchiyama K. Functional evaluations comparing the double-tract method and the jejunal interposition method following laparoscopic proximal gastrectomy for gastric cancer: an investigation including laparoscopic total gastrectomy. Surg Today. 2019;49(1):38-48.

31. Ahn SH, Jung DH, Son SY, Lee CM, Park DJ, Kim HH. Laparoscopic double-tract proximal gastrectomy for proximal early gastric cancer. Gastric cancer: official journal of the International Gastric Cancer Association and the Japanese Gastric Cancer Association. 2014;17(3):562 - 70.

32. Yamashita K, Iwatsuki M, Koga Y, Toihata T, Kiyozumi Y, Kuroda D, Eto K, Hiyoshi Y, Iwagami S, Baba Y, Miyamoto Y, Yoshida N, Baba H. Preservation of physiological passage through the remnant 
stomach prevents postoperative malnutrition after proximal gastrectomy with double tract reconstruction. Surgery today. 2019;49(9):748 - 54.

33. Takiguchi $N$, Takahashi M, Ikeda M, Inagawa S, Ueda S, Nobuoka T, Ota M, Iwasaki Y, Uchida N, Kodera Y, Nakada K. Long-term quality-of-life comparison of total gastrectomy and proximal gastrectomy by postgastrectomy syndrome assessment scale (PGSAS-45): a nationwide multiinstitutional study. Gastric cancer: official journal of the International Gastric Cancer Association and the Japanese Gastric Cancer Association. 2015;18(2):407 - 16.

34. An JY, Youn HG, Choi MG, Noh JH, Sohn TS, Kim S. The diffificult choice between total and proximal gastrectomy in proximal early gastric cancer. Am J Surg. 2008;196(4):587 - 91.

35. Ahn SH, Lee JH, Park DJ, Kim HH. Comparative study of clinical outcomes between laparoscopyassisted proximal gastrectomy (LAPG) and laparoscopy-assisted total gastrectomy (LATG) for proximal gastric cancer. Gastric cancer: official journal of the International Gastric Cancer Association and the Japanese Gastric Cancer Association. 2013;16(3):282-9.

36. Ronellenfitsch U, Najmeh S, Andalib A, Perera RM, Rousseau MC, Mulder DS, Ferri LE. Functional outcomes and quality of life after proximal gastrectomy with esophagogastrostomy using a narrow gastric conduit. Annals of surgical oncology. 2015;22(3):772-9.

\section{Figures}




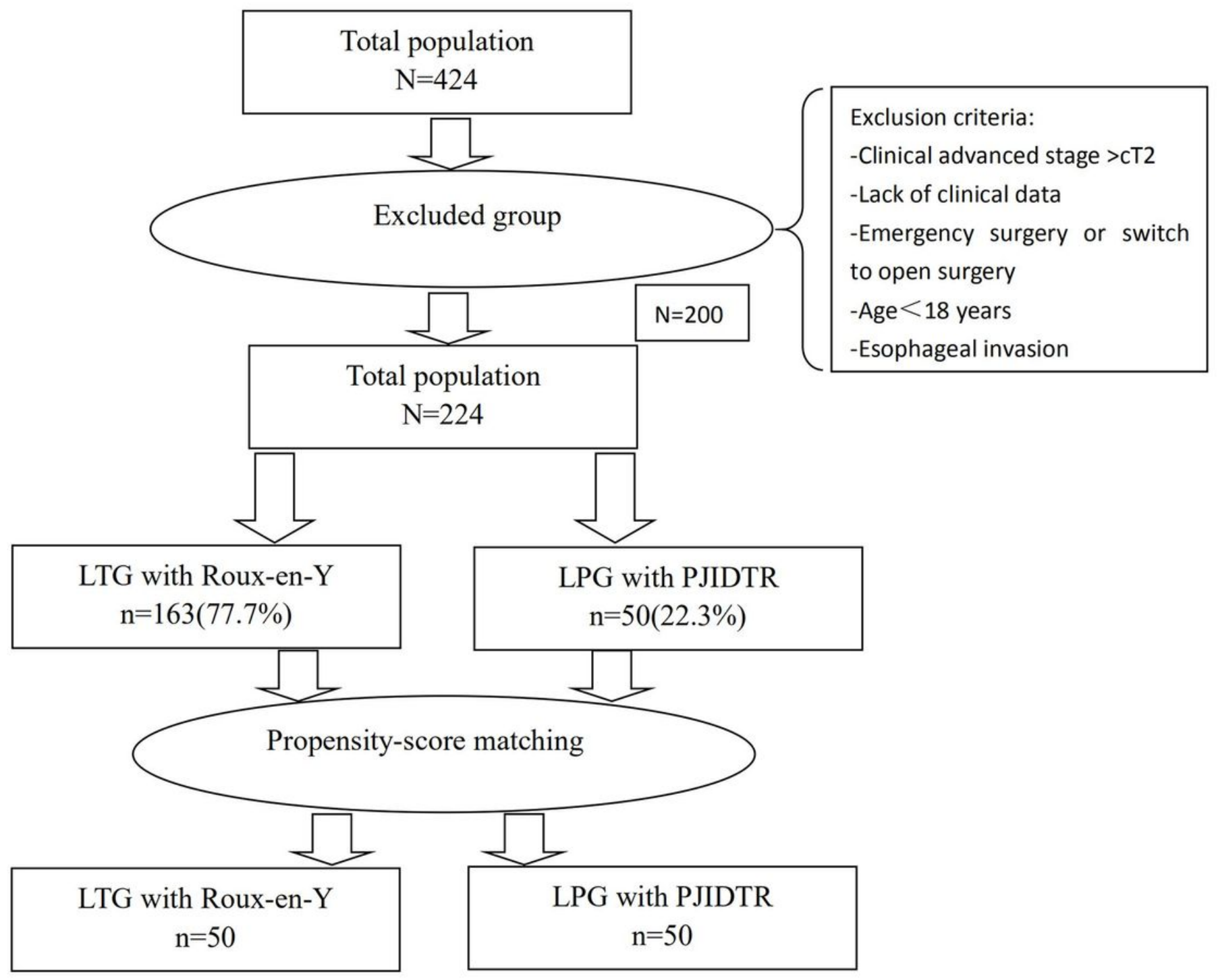

\section{Figure 1}

Flow chart of patient selection and propensity score matching. The 50 patients who underwent laparoscopic proximal gastrectomy (LPG) with piggyback jejunal interposition double-tract reconstruction (PJIDTR) were matched to 50 patients who underwent laparoscopic total gastrectomy (LTG) with Rouxen-Y in terms of age, sex, body mass index, differentiation, pathological stage, and American Society of Anesthesiologists (ASA) score. 


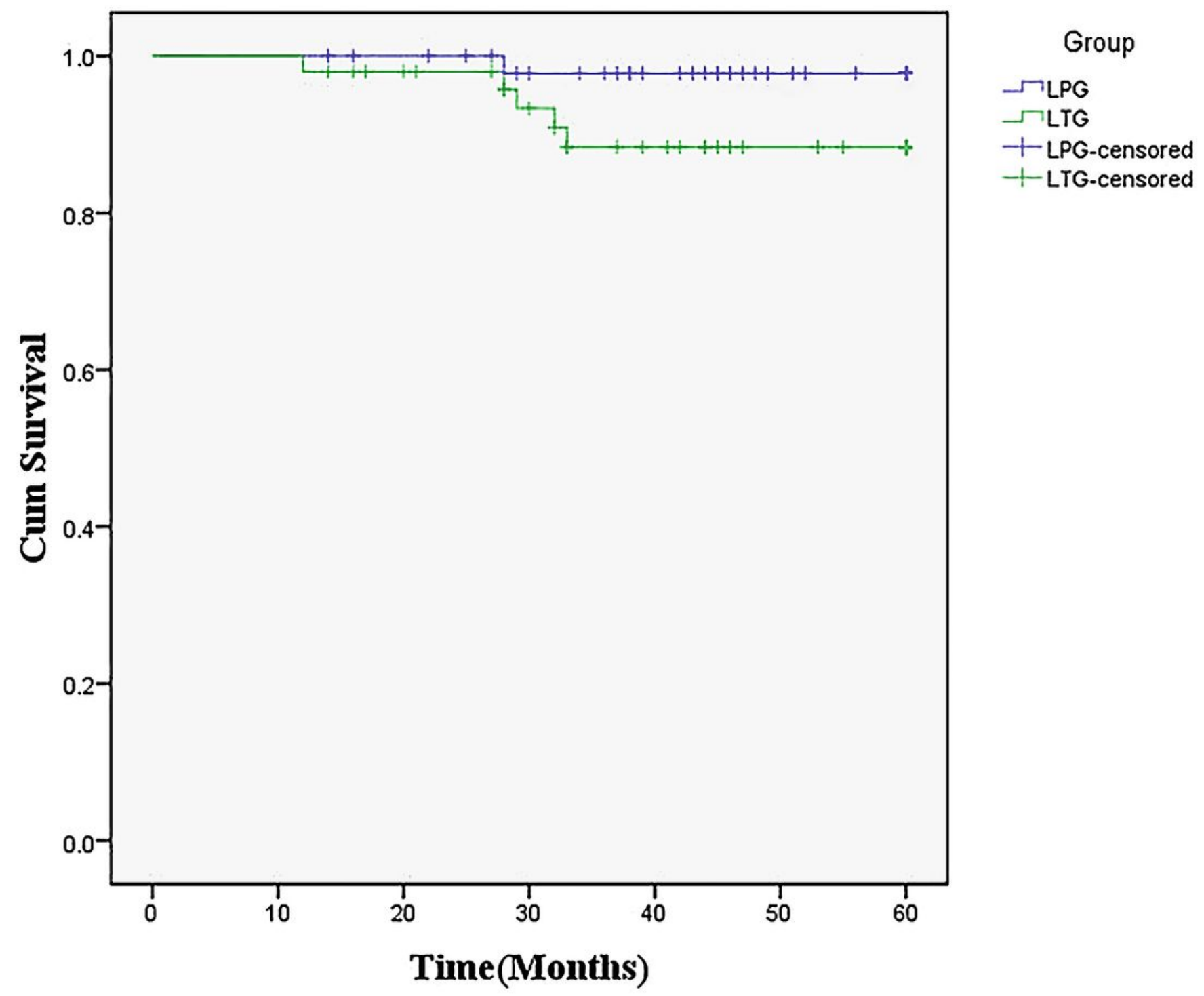

Figure 2

Comparison of the propensity score-matched laparoscopic proximal gastrectomy (LPG) and laparoscopic total gastrectomy (LTG) groups in terms of overall survival rate. The two groups differed significantly in overall survival ( $p$ value $=0.08$ ), as determined using the log-rank test. 